J. Korean Math. Soc. 51 (2014), No. 4, pp. 751-771

http://dx.doi.org/10.4134/JKMS.2014.51.4.751

\title{
STRUCTURE OF IDEMPOTENTS IN RINGS WITHOUT IDENTITY
}

\author{
Nam Kyun Kim, Yang Lee, and Yeonsook Seo
}

\begin{abstract}
We study the structure of idempotents in polynomial rings power series rings, concentrating in the case of rings without identity. In the procedure we introduce right Insertion-of-Idempotents-Property (simply, right IIP) and right Idempotent-Reversible (simply, right IR) as generalizations of Abelian rings. It is proved that these two ring properties pass to power series rings and polynomial rings. It is also shown that $\pi$-regular rings are strongly $\pi$-regular when they are right IIP or right IR. Next the noncommutative right IR rings, right IIP rings, and Abelian rings of minimal order are completely determined up to isomorphism. These results lead to methods to construct such kinds of noncommutative rings appropriate for the situations occurred naturally in studying standard ring theoretic properties.
\end{abstract}

\section{Definitions and notations}

Throughout this paper, $R$ denotes an associative ring without identity, unless otherwise stated. Denote the $n$ by $n$ full (resp., upper triangular) matrix ring over $R$ by $\operatorname{Mat}_{n}(R)$ (resp., $U_{n}(R)$ ). We let $e_{i j}$ denote the usual matrix units with 1 in the $(i, j)$-position and zeros elsewhere, if the base ring has identity 1. Denote $\left\{\left(a_{i j}\right) \in U_{n}(R) \mid\right.$ the diagonal entries of $\left(a_{i j}\right)$ are all equal $\}$ by $D_{n}(R)$. $\mathbb{Z}_{n}$ denotes the ring of integers modulo $n . G F\left(p^{n}\right)$ denotes the Galois field of order $p^{n} . J(R)$ denotes the Jacobson radical of $R$. $\mid$ denotes the cardinality. The characteristic of $R$ is denoted by char $R$, and $|a|$ denotes the order of $a \in R$ in the additive subgroup of $R$ generated by $a . R^{+}$means the additive Abelian group $(R,+)$. The polynomial ring with an indeterminate $x$ over $R$ is denoted by $R[x]$. While speaking about minimal ring in a certain class of rings, we refer to a ring with minimal order for rings in that class, due to Xue [21]. The notation $(S)$ stands for the two-sided ideal of $R$ generated by $\emptyset \neq S \subseteq R$, and we also write $\left(a_{1}, \ldots, a_{n}\right)$ in place of $(S)$ for simplicity when $S=\left\{a_{1}, \ldots, a_{n}\right\}$.

A ring is called Abelian if every idempotent is central. The zero in a nil ring is the only idempotent and so every nil ring is Abelian. The class of Abelian

Received November 2, 2013.

2010 Mathematics Subject Classification. 16P10, $16 \mathrm{U} 80$.

Key words and phrases. idempotent, right IIP ring, right IR ring, Abelian ring. 
rings contains reduced rings (i.e., rings without nonzero nilpotent elements) and commutative rings. Another generalization of both reduced rings and commutative rings is Insertion-of-Factors-Property (simply IFP) which was introduced by Bell [4]. Due to Bell, a ring $R$ is usually called IFP if $a b=0$ implies $a R b=0$ for $a, b \in R$. Shin [20] used the term SI for the IFP, while Narbonne [19] used semicommutative in place of the IFP. In fact reduced rings are easily shown to be IFP. It is also straightforward to check that every IFP ring is Abelian when it has an identity. However this result is no longer valid for rings without identity as we see in Section 2.

\section{Right IIP rings and right IR rings}

In this section we observe some interesting ring-theoretic generalizations of Abelian rings. We introduce the concepts of right IIP rings and right IR rings. The minimal non-Abelian one-sided IIP (IR) rings will be completely determined, up to isomorphism. It will be also shown that one-sided IR condition passes to power series rings and polynomial rings.

Lemma 2.1. For a ring $R$ with identity the following conditions are equivalent:

(1) $R$ is Abelian;

(2) If $a b e=0$ for $a, b, e^{2}=e \in R$, then $a e b=0$;

(3) If eab=0 for $a, b, e^{2}=e \in R$, then $a e b=0$;

(4) If $a e=0$ for $a, e^{2}=e \in R$, then $e a=0$;

(5) If $e a=0$ for $a, e^{2}=e \in R$, then $a e=0$.

Proof. $(1) \Rightarrow(2),(2) \Rightarrow(4),(1) \Rightarrow(3)$, and $(3) \Rightarrow(5)$ are obvious.

Let $e^{2}=e, r \in R$. Then $r(1-e) e=0$ and $r e(1-e)=0$. If $R$ satisfies the condition (4), then $\operatorname{er}(1-e)=0$ and $(1-e) r e=0$. These yield $r e=e r$, showing $(4) \Rightarrow(1)$. The proof of $(5) \Rightarrow(1)$ is similar.

In this paper we consider the conditions of Lemma 2.1 in rings without identity, and observe the structure of rings satisfying the conditions.

Definition 2.2. (1) A ring $R$ is said to satisfy right (resp. left) Insertionof-Idempotents-Property (simply, called right (resp. left) IIP) if it satisfies the condition (2) (resp. (3)) of Lemma 2.1.

(2) A ring is called right (resp. left) Idempotent-Reversible (simply, IR) if it satisfies the condition (4) (resp. (5)) in Lemma 2.1.

The class of one-sided IIP rings contains both Abelian rings and nil rings, and right (resp. left) IIP rings are right (resp. left) IR by Lemma 2.4 below. A ring is both left and right IIP if and only if it is both left and right IR if and only if it is Abelian by Proposition 2.8 to follow.

This Insertion-of-Idempotents-Property is not left-right symmetric as illustrated by the following example. 
Example 2.3. (1) Let $F$ be a field and $F\langle a, b\rangle$ the free algebra with noncommuting two indeterminates $a, b$ over $F$. Define

$$
A=\{f \in F\langle a, b\rangle \mid \text { the constant term of } f \text { is zero }\}
$$

and $I$ the ideal of $A$ generated by

$$
a b, a^{2}-a, b^{2}
$$

Set $R=A / I$ and identify the elements in $A$ with their images in $R$ for simplicity. Then $a^{2}=a$ and $a b=0=b^{2}$ in $R$. Since $a b a=0$ (with $a^{2}=a$ ) but $b a a=b a \neq 0, R$ is not left IIP.

We will show that $R$ is right IIP. By the construction of $R$, every element $f \in R$ is of the form

$$
f=\alpha_{1} a+\alpha_{2} b a+\alpha_{3} b
$$

where $\alpha_{1}, \alpha_{2}, \alpha_{3} \in F$.

Suppose $f^{2}=f$ for $f=\alpha_{1} a+\alpha_{2} b a+\alpha_{3} b$. Then

$$
\begin{aligned}
& \alpha_{1}^{2} a=\alpha_{1} a ; \\
& \alpha_{2} b a=\alpha_{1}\left(\alpha_{2}+\alpha_{3}\right) b a ; \\
& \alpha_{3} b=0 .
\end{aligned}
$$

Eq. (3) implies $\alpha_{3}=0$, entailing $\alpha_{2} b a=\alpha_{1} \alpha_{2} b a$. Eq. (1) implies $\alpha_{1}^{2}=\alpha_{1}$, and hence $\alpha_{1}=0$ or $\alpha_{1}=1$.

Assume $\alpha_{1}=0$. Then Eq. (2) implies $\alpha_{2} b a=0$ and so $\alpha_{2}=0$. This yields $f=0$.

Assume $\alpha_{1}=1$. Then $\alpha_{2} b a=\alpha_{2} b a$ since $\alpha_{2} b a=\alpha_{1} \alpha_{2} b a$. This yields $f=a+\alpha_{2} b a$.

Therefore the set of all idempotents in $R$ is

$$
E=\{0, a+\gamma b a \mid \gamma \in F\} .
$$

Now let $f=\alpha_{1} a+\alpha_{2} b a+\alpha_{3} b$ and $g=\beta_{1} a+\beta_{2} b a+\beta_{3} b$ for $\alpha_{i}, \beta_{i} \in F$ for $i=1,2,3$. Then, for $e=a+\gamma b a \in E$ with $\gamma \in F$, we have

$$
\begin{aligned}
f g e=f g(a+\gamma b a) & =\left(\alpha_{1} \beta_{1} a+\left(\alpha_{2} \beta_{1}+\alpha_{3} \beta_{1}\right) b a\right)(a+\gamma b a) \\
& =\alpha_{1} \beta_{1} a+\left(\alpha_{2} \beta_{1}+\alpha_{3} \beta_{1}\right) b a \\
& =\left(\alpha_{1} a+\alpha_{2} b a+\alpha_{3} b\right) \beta_{1} a \\
& =\left(\alpha_{1} a+\alpha_{2} b a+\alpha_{3} b\right) a \beta_{1} a \\
& =\left(\alpha_{1} a+\alpha_{2} b a+\alpha_{3} b\right) a\left(\beta_{1} a+\beta_{2} b a+\beta_{3} b\right) \\
& =\left(\alpha_{1} a+\alpha_{2} b a+\alpha_{3} b\right)(a+\gamma b a)\left(\beta_{1} a+\beta_{2} b a+\beta_{3} b\right) \\
& =f(a+\gamma b a) g=f e g .
\end{aligned}
$$

Then $f g e=0$ implies $f e g=0$ for $e^{2}=e \in R$. Therefore $R$ is right IIP.

(2) Let $A$ be the same ring as in (1). Let $J$ be the ideal of $A$ generated by

$$
b a, a^{2}-a, b^{2} .
$$


Then the ring $R=A / J$ is not right IIP but left IIP by a similar computation to $(1)$.

(3) Let $D$ be a domain with identity and $R=\left(\begin{array}{cc}D & D \\ 0 & 0\end{array}\right)$ a subring of $U_{2}(D)$. Note that every nonzero idempotent is of the form $\left(\begin{array}{cc}1 & \alpha \\ 0 & 0\end{array}\right)$ with $\alpha \in D$. Consider

$$
x=\left(\begin{array}{ll}
a & b \\
0 & 0
\end{array}\right), y=\left(\begin{array}{ll}
c & d \\
0 & 0
\end{array}\right), e=\left(\begin{array}{ll}
1 & \alpha \\
0 & 0
\end{array}\right) \in R .
$$

Then exy $=\left(\begin{array}{cc}a c & a d \\ 0 & 0\end{array}\right)=x e y$, but

$$
\text { xye }=\left(\begin{array}{cc}
a c & a c \alpha \\
0 & 0
\end{array}\right) \neq\left(\begin{array}{cc}
a c & a d \\
0 & 0
\end{array}\right)=x e y
$$

when $a c \alpha \neq a d$ (e.g. $a=c=\alpha=1, d=0$ ). Thus $R$ is left IIP but not right IIP.

(4) Let $D$ be a domain with identity and $S=\left(\begin{array}{ll}0 & D \\ 0 & D\end{array}\right)$ a subring of $U_{2}(D)$. Then $S$ is right IIP but not left IIP through a similar computation as in (3), noting that every nonzero idempotent is of the form $\left(\begin{array}{ll}0 & \beta \\ 0 & 1\end{array}\right)$ with $\beta \in D$.

As we see in Example 2.3, one-sided IIP rings need not be Abelian when they do not have identity.

Lemma 2.4. If $R$ is a right (resp. left) IIP ring, then $R$ is right (resp. left) $I R$.

Proof. Let $R$ be right (resp. left) IIP. Suppose $a e=0$ (resp. $e a=0$ ) for $a, e^{2}=e \in R$. Then $e a e=0$, and since $R$ is right (resp. left) IIP we get $e a=e e a=0$ (resp. $a e=a e e=0)$.

Regularity, $\pi$-regularity, and strong $\pi$-regularity have important roles in ring theory and module theory. A ring $R$ is said to be $\pi$-regular if for every $x \in R$ there exists a positive integer $n$, depending on $x$, such that $x^{n} \in x^{n} R x^{n}$. A ring $R$ is said to be strongly $\pi$-regular if for every $x \in R$ there exists a positive integer $n$, depending on $x$, such that $x^{n} \in R x^{n+1}$. Strong $\pi$-regularity is leftright symmetric by Dischinger [7]. A ring $R$ is said to be (von Neumann) regular if $x \in x R x$ for every $x \in R$. Regular rings are clearly $\pi$-regular but the converse need not hold as can be seen by $\mathbb{Z}_{4}$. Strongly $\pi$-regular rings are $\pi$-regular by Azumaya [3]. For a division ring $D$ and a right $D$-module $V$, it is well-known that the endomorphism ring of $V$ over $D$ is strongly $\pi$-regular if and only if $V$ is finite dimensional over $D$. So if $V$ is infinite dimensional over $D$, then the endomorphism ring of $V$ over $D$ is regular (hence $\pi$-regular) but not strongly $\pi$-regular. Abelian $\pi$-regular rings are clearly strongly $\pi$-regular.

Proposition 2.5. A right IR $\pi$-regular ring is strongly $\pi$-regular.

Proof. Let $R$ be a right IR $\pi$-regular ring and $x \in R$. Since $R$ is $\pi$-regular, there exist $y \in R$ and a positive integer $n$ such that $x^{n}=x^{n} y x^{n}$. Here we claim $x^{n}=x^{2 n} y$, implying that $R$ is strongly $\pi$-regular. We first have $\left(x^{n}-\right.$ 
$\left.x^{2 n} y\right) x^{n} y=x^{n} x^{n} y-x^{n}\left(x^{n} y x^{n}\right) y=x^{n} x^{n} y-x^{n}\left(x^{n}\right) y=0$. Since $\left(x^{n} y\right)^{2}=x^{n} y$ and $R$ is right IR, we get $0=x^{n} y\left(x^{n}-x^{2 n} y\right)=x^{n}-x^{2 n} y$ and $x^{n}=x^{2 n} y$.

Note. (1) The idempotent-reversible condition is also not left-right symmetric by Example 2.3(1,2). Letting $R$ be the ring in Example 2.3(1) (resp. Example $2.3(2)$ ), we have that $R$ is right (resp. left) IIP (hence right (resp. left) IR) and that $R$ is not left (resp. right) IR since $a b=0$ but $b a \neq 0$ (resp. $b a=0$ but $a b \neq 0$ ), where $a^{2}=a, b \in R$.

(2) The ring in Example 2.3(3) (resp. Example 2.3(4)) is not right (resp. not left) IR as can be seen by $e_{12} e_{11}=0 \neq e_{11} e_{12}=e_{12}\left(\right.$ resp. $\left.e_{12} e_{22} \neq 0=e_{22} e_{12}\right)$.

The following examples illustrate that the idempotent-reversible condition is not left-right symmetric, and that right IR rings need not be right IIP.

Example 2.6. (1) There exists a right IR ring that is not right IIP. Let $F$ be a field and $F\langle a, b\rangle$ the free algebra with noncommuting indeterminates $a, b$ over $F$. Define

$$
A=\{f \in F\langle a, b\rangle \mid \text { the constant term of } f \text { is zero }\}
$$

and $J$ the ideal of $A$ generated by

$$
a b, a^{2}-a .
$$

Set $R=A / J$ and identify the elements in $A$ with their images in $R$ for simplicity. Then $a^{2}=a$ and $a b=0$ in $R$. Since $a b=0$ (with $a^{2}=a$ ) but $b a \neq 0$, $R$ is not left IR.

We will show that $R$ is right IR but not right IIP. By the construction of $R$, every element $f \in R$ is of the form

$$
f=\alpha a+f_{1} a+f_{2}
$$

where $\alpha \in F$ and $f_{1}, f_{2} \in b F[b]$.

Suppose $f^{2}=f$ for $f=\alpha a+f_{1} a+f_{2} \in R$. Then

$$
\begin{aligned}
& \alpha^{2} a=\alpha a ; \\
& f_{1} a=\left(\alpha f_{1}+\alpha f_{2}+f_{2} f_{1}\right) a ; \\
& f_{2}=f_{2}^{2} .
\end{aligned}
$$

Eq. (3) implies $f_{2}=0$, entailing $f_{1} a=\alpha f_{1} a$ from Eq. (2). Next Eq. (1) implies $\alpha^{2}=\alpha$, and hence $\alpha=0$ or $\alpha=1$.

Assume $\alpha=0$. Then $f_{1} a=\alpha f_{1} a=0$ and so $f_{1}=0$, leading to $f=0$.

Assume $\alpha=1$. Then $f_{1} a=\alpha f_{1} a=f_{1} a$ and so $f=a+f_{1} a$.

Therefore the set of all idempotents in $R$ is

$$
E=\{0, a+k a \mid k \in b F[b]\} .
$$

Now let $f=\alpha a+f_{1} a+f_{2} \in R$ for $\alpha \in F$ and $f_{i} \in b F[b]$ for $i=1,2$. If $e=0$, then clearly $f e=0=e f$. If $f e=0$ for $e=a+k a \in E$ with $k \in b F[b]$, then we have

$$
0=\left(\alpha a+f_{1} a+f_{2}\right)(a+k a)=\alpha a+\left(f_{1}+f_{2}+f_{2} k\right) a
$$


and so $\alpha=0$, this leads to $f=f_{1} a+f_{2}$. This yields

$$
e f=(a+k a)\left(f_{1} a+f_{2}\right)=0
$$

by the construction of $J$. Thus $R$ is right IR.

Let

$$
x=b a-b, y=a, e=a+b a .
$$

Then $x y e=0$ but $x e y=(b a-b)(a+b a) a=-b^{2} a \neq 0$; hence $R$ is not right IIP.

(2) Let $A$ be the same ring as in (1). Let $K$ be the ideal of $A$ generated by

$$
b a, a^{2}-a \text {. }
$$

Then the ring $R=A / K$ is left IR (and not left IIP) but not right IR by a similar computation to (1).

Due to Lambek [17], a ring $R$ is called symmetric if $r s t=0$ implies $r t s=0$ for all $r, s, t \in R$. Anderson-Camillo [1] used the term $Z C_{3}$ for symmetric rings. Lambek proved, in [17, Proposition 1], that a ring $R$ is symmetric if and only if $r_{\sigma(1)} r_{\sigma(2)} \cdots r_{\sigma(n)}=0$ for any permutation $\sigma$ of the set $\{1,2, \ldots, n\}$ when $r_{1} r_{2} \cdots r_{n}=0$ for any positive integer $n$ and $r_{i} \in R$. Anderson-Camillo also obtained this result independently in [1, Theorem I.1]. We will use this result freely. According to Cohn [6], a ring $R$ is called reversible if $a b=0$ implies $b a=0$ for $a, b \in R$. Anderson-Camillo [1] used the term $Z C_{2}$ for reversible rings. Essential properties of symmetric rings and reversible rings are developed in related literature. It is evident that commutative rings are both symmetric and reversible. Reduced rings are symmetric by [1, Theorem I.3] or $[17$, Proposition 1], and there are many kinds of non-reduced commutative rings.

If $R$ is a symmetric ring with identity, then $R$ is clearly reversible (but the converse need not hold by [1, Examples I.5] or [18, Examples 5 and 7]); however for rings without identity this implication is no longer valid as we see in the following example.

Example 2.7. (1) Let $A$ be a ring with identity and $R=\left\{\left(\begin{array}{lll}0 & a & b \\ 0 & 0 & c \\ 0 & 0 & 0\end{array}\right) \mid a, b, c \in A\right\}$ a subring of $U_{3}(A)$. Since $R^{3}=0, R$ is clearly symmetric. But since $e_{23} e_{12}=0$ and $e_{12} e_{23}=e_{13} \neq 0, R$ is not reversible.

(2) The construction is due to [18, Example 1]. Let $S=\{a, b\}$ be the semigroup with multiplication $a^{2}=a b=a, b^{2}=b a=b$. Put $R=\mathbb{Z}_{2} S$, which is a four-element semigroup ring. Then $a(a+b)=a^{2}+a b=2 a=0$ but $(a+b) a=a^{2}+b a=a+b \neq 0$; hence $R$ is not reversible. Since $x y z=x z y$ for all $x, y, z \in R, R$ is symmetric.

Recall that any symmetric ring is Abelian when it has an identity. But a symmetric ring, without identity, need not be Abelian as can be seen by the relations $a^{2}=a, b^{2}=b, a=a b \neq b a=b$ in Example 2.7(2). The class of Abelian rings contains reversible rings as we see in the following proposition. 
Proposition 2.8. Let $R$ be a ring.

(1) $R$ is right (resp. left) IR if and only if er $=$ ere (resp. re =ere) for every $e^{2}=e \in R$ and $r \in R$.

(2) If $R$ is right (resp. left) IIP, then er = ere (resp. re = ere) for every $e^{2}=e \in R$ and $r \in R$.

(3) If $R$ is reversible, then $R$ is Abelian.

Proof. (1) Let $R$ be right (resp. left) IR, and $r, e^{2}=e \in R$. Then $e(e r-$ $r e) e=0$. Since $R$ is right (resp. left) IR, $0=e e(e r-r e)=e r-e r e$ (resp. $0=(e r-r e) e e=e r e-r e)$. This implies $e r=e r e($ resp. $r e=e r e)$.

Conversely, suppose that $e r=e r e$ for every $r, e^{2}=e \in R$. Let $a f=0$ for $a, f^{2}=f \in R$. Then $f a=f a f=0$. The computation for the left case is similar.

(2) is obtained from Lemma 2.4 and (1).

(3) It is obvious that reversible rings are left and right IR. So (3) is an immediate consequence of (1).

The following corollary is obtained immediately from Proposition 2.8(1), (2).

Corollary 2.9. Let $R$ be a ring. Then $R$ is both left and right IR if and only if $R$ is Abelian if and only if $R$ is both left and right IIP.

Note that both symmetric rings and reversible rings are IFP. There exist many kinds of Abelian rings which are not IFP (hence are neither reversible nor symmetric) as we see in the following example.

Example 2.10. (1) Let $A$ be a ring with identity and

$$
R=\left\{\left(\begin{array}{cccc}
0 & a & b & c \\
0 & 0 & d & e \\
0 & 0 & 0 & f \\
0 & 0 & 0 & 0
\end{array}\right) \mid a, b, c, d, e, f \in A\right\}
$$

a subring of $U_{4}(A)$. Since $R^{4}=0,0$ is the only idempotent and so $R$ is clearly Abelian. But $e_{23} e_{12}=0, e_{12} e_{23}=e_{13} \neq 0$, and $e_{12} e_{34} e_{23}=0, e_{12} e_{23} e_{34}=$ $e_{14} \neq 0$. So $R$ is not IFP and so is neither reversible nor symmetric.

(2) Let $D$ be a domain with identity and $A=D \oplus D$. Define

$$
R=\left\{\left(\begin{array}{cccc}
\alpha & a & b & c \\
0 & \alpha & d & e \\
0 & 0 & \alpha & f \\
0 & 0 & 0 & \alpha
\end{array}\right) \mid \alpha \in D \oplus 0 \text { and } a, b, c, d, e, f \in A\right\}
$$

as a subring of $U_{4}(A)$. Then $R$ is Abelian by [12, Lemma 2], but $R$ is not IFP by the same calculation as in (1). 
(3) Let $A$ be an Abelian ring with identity and

$$
R=\left\{\left(\begin{array}{cccc}
\beta & a & b & c \\
0 & \beta & d & e \\
0 & 0 & \beta & f \\
0 & 0 & 0 & \beta
\end{array}\right) \mid \beta, a, b, c, d, e, f \in A\right\}
$$

a subring, of $U_{4}(A)$, with identity. Then $R$ is Abelian by [12, Lemma 2], but $R$ is not IFP by the same calculation as in (1).

(4) The construction is due to [14, Example 2.1]. Let $A=\mathbb{Z}_{2}\left\langle a_{0}, a_{1}, a_{2}, b_{0}, b_{1}\right.$, $\left.b_{2}\right\rangle$ be the free algebra with noncommuting indeterminates $a_{0}, a_{1}, a_{2}, b_{0}, b_{1}, b_{2}$ over $\mathbb{Z}_{2}$. Next define $B$ as the subalgebra of $A$ of polynomials with zero constant terms. Consider an ideal of the $\operatorname{ring} B$, say $I$, generated by the following elements:

$$
\begin{gathered}
a_{0} b_{0}, a_{0} b_{1}+a_{1} b_{0}, a_{0} b_{2}+a_{1} b_{1}+a_{2} b_{0}, a_{1} b_{2}+a_{2} b_{1}, a_{2} b_{2}, a_{0} r b_{0}, a_{2} r b_{2}, \\
b_{0} a_{0}, b_{0} a_{1}+b_{1} a_{0}, b_{0} a_{2}+b_{1} a_{1}+b_{2} a_{0}, b_{1} a_{2}+b_{2} a_{1}, b_{2} a_{2}, b_{0} r a_{0}, b_{2} r a_{2}, \\
\left(a_{0}+a_{1}+a_{2}\right) r\left(b_{0}+b_{1}+b_{2}\right),\left(b_{0}+b_{1}+b_{2}\right) r\left(a_{0}+a_{1}+a_{2}\right), \text { and, } r_{1} r_{2} r_{3} r_{4},
\end{gathered}
$$

where $r, r_{1}, r_{2}, r_{3}, r_{4} \in B$. Set $R=B / I$. Then $R$ is a ring which is both reversible and symmetric by the calculation in [11, Example 3.1]. But $R[x]$ is not IFP (hence is neither reversible nor symmetric) as can be seen by the products $\left(a_{0}+a_{1} x+a_{2} x^{2}\right)\left(b_{0}+b_{1} x+b_{2} x^{2}\right)=0$ and $\left(a_{0}+a_{1} x+a_{2} x^{2}\right) R\left(b_{0}+\right.$ $\left.b_{1} x+b_{2} x^{2}\right) \neq 0$. Note that $R[x]$ is Abelian by [13, Lemma 8] since $R$ is Abelian by Proposition $2.8(3)$. Notice that $A / I$, with identity, also satisfies the same properties as $R$.

We also see the relations among the concepts above for rings without identity in the following example.

Example 2.11. (1) Let $D$ be a domain and $R=\left(\begin{array}{cc}D & D \\ 0 & 0\end{array}\right)$. Let $0 \neq x=$ $\left(\begin{array}{ll}a & b \\ 0 & 0\end{array}\right), 0 \neq y=\left(\begin{array}{cc}c & d \\ 0 & 0\end{array}\right) \in R$. If $x y=0$, then $a=0$. Whence for any $z=\left(\begin{array}{ll}e & f \\ 0 & 0\end{array}\right) \in R$ we also get $x z y=0$. So $R$ is IFP, but $R$ is non-Abelian as can be seen by the idempotent $e_{11}$. A similar calculation shows that $\left(\begin{array}{ll}0 & D \\ 0 & D\end{array}\right)$ is also IFP but nonAbelian. These non-Abelian IFP rings are neither reversible nor symmetric by Proposition 2.8(3).

(2) Let $D$ be a domain. Then $A=\left(\begin{array}{ll}D & D \\ 0 & 0\end{array}\right)$ and $B=\left(\begin{array}{ll}0 & D \\ 0 & D\end{array}\right)$ are both IFP by the argument in (1), but $A$ is not right IR (hence not right IIP) and $B$ is not left IR (hence not left IIP) by Lemma 2.4.

(3) An IFP ring, with identity, need not reversible by the subring

$$
R=\left\{\left(\begin{array}{ccc}
a & b & c \\
0 & a & d \\
0 & 0 & a
\end{array}\right) \mid a, b, c, d \in A\right\}
$$

of $U_{3}(A)$, where $A$ is a reduced ring. In fact, $R$ is IFP by [14, Proposition 1.2], but it is not reversible since $e_{12} e_{23}=e_{13} \neq 0=e_{23} e_{12}$. 
(4) Let $R$ be any ring in Example 2.10(1,2,3). Then $R$ is Abelian (hence left and right IIP) by the arguments in Example $2.10(1,2,3)$. However $R$ is not IFP by [14, Example 1.3]. In [14, Example 1.3], we see that $\left(e_{12}-e_{13}\right)\left(e_{24}+e_{34}\right)=0$ but $\left(e_{12}-e_{13}\right) e_{23}\left(e_{24}+e_{34}\right)=e_{14}$.

There exist many regular rings without identity as can be seen by the direct sum of infinitely many regular rings with identity. For regular rings, previously mentioned ring properties are all equivalent as we see in the following theorem.

Theorem 2.12. Given a regular ring $R$, the following conditions are equivalent:

(1) $R$ is reduced;

(2) $R$ is symmetric;

(3) $R$ is reversible;

(4) $R$ is IFP;

(5) $R$ is Abelian;

(6) $R$ is right IIP;

(7) $R$ is left IIP;

(8) $R$ is right IR;

(9) $R$ is left IR.

Proof. We have obviously the implications $(1) \Rightarrow(2),(1) \Rightarrow(3),(2) \Rightarrow(4)$, $(3) \Rightarrow(4),(5) \Rightarrow(6)$, and $(5) \Rightarrow(7)$. The implications $(2) \Rightarrow(5)$ and $(3) \Rightarrow(5)$ are obtained by Proposition 2.8(3). If $R$ is Abelian and $a^{2}=0$ for $a \in R$, then $0=a^{2}=a^{2} b=a a b=a b a=a$ and so $R$ is reduced, where $a=a b a$ for some $b \in R$ (by the regularity of $R$ ). Thus the conditions (1), (2), (3), and (5) are always equivalent when $R$ is regular. The implications $(6) \Rightarrow(8)$ and $(7) \Rightarrow(9)$ are obtained by Lemma 2.4 .

When $R$ has an identity then $(4) \Rightarrow(5)$ is obvious. So if $R$ has an identity, then the equivalences are obtained by Lemmas 2.1 and 2.4. So we assume that $R$ does not have an identity. So it suffices to show that $(8) \Rightarrow(5),(9) \Rightarrow(5)$, and $(4) \Rightarrow(5)$.

$(8) \Rightarrow(5)$ : Let $R$ be right IR. Assume on the contrary that there exist $r, e^{2}=e \in R$ with $r e-e r \neq 0$. Let $x=r e-e r$. Then $e r e=e r$ by Proposition $2.8(1)$, entailing $e x=0$. Since $R$ is regular, there exists $y \in R$ such that $x=x y x$. Then

$$
0=e x=x e x y=x y x e=x e .
$$

Now $e x=0=x e$ gives $e r=e r e=r e$, a contradiction. Thus $R$ is Abelian. The proof of $(9) \Rightarrow(5)$ is similar.

$(4) \Rightarrow(5)$ : Let $R$ be IFP. Assume on the contrary that there exist $r, e^{2}=$ $e \in R$ with $r e-e r \neq 0$. Let $y=e r e-r e$, then $e y=0$. Since $R$ is regular, there exists $z \in R$ such that ye=yezye. Then

$$
0=e y=e z y=y e z y e=y e
$$


leading to $e r e=r e$. Next let $y_{1}=e r e-e r$, then $y_{1} e=0$. Since $R$ is regular, there exists $z_{1} \in R$ such that $e y_{1}=e y_{1} z_{1} e y_{1}$. Then

$$
0=y_{1} e=y_{1} z_{1} e=e y_{1} z_{1} e y_{1}=e y_{1},
$$

hence $e r e=e r$. Now we get $r e=e r e=e r$, a contradiction.

As we see in Example 2.11, IFP rings without identity need not be Abelian and so the proofs of directions (e.g., $(4) \Rightarrow(5)$ ) in Theorem 2.12 are necessary.

Regular rings are clearly $\pi$-regular, and there exist many kinds of $\pi$-regular rings that are not regular rings. Based on Theorem 2.12, one may conjecture that $\pi$-regular right IIP rings are also Abelian. But the ring $S=\left(\begin{array}{ll}0 & D \\ 0 & D\end{array}\right)(D$ : a division ring) is a non-Abelian right IIP ring by Example 2.3(4). A quick calculation shows that $S$ is not regular but $\pi$-regular.

In the following arguments we examine various kinds of ring extensions on the right IIP and right IR conditions. Quick calculations reveal the following lemma.

Lemma 2.13. (1) The class of right IIP rings is closed under subrings, direct sums, and direct products.

(2) The class of right IR rings is closed under subrings, direct sums, and direct products.

(3) The class of Abelian rings is closed under subrings, direct sums, and direct products.

We first compute the case of polynomial rings and power series rings. Given a ring $R$ the power series ring, with an indeterminate $x$ over $R$, is denoted by $R[[x]]$.

Theorem 2.14. Let $R$ be a ring. Then we have the following results:

(1) $R$ is right $I R$ if and only if so is $R[[x]]$.

(2) $R$ is right $I R$ if and only if so is $R[x]$.

Proof. (1) It suffices to show the necessity. Let $R$ be right IR. Suppose that $f(x) e(x)=0$ for $f(x)=\sum_{i=0}^{\infty} a_{i} x^{i}, e(x)^{2}=e(x)=\sum_{j=0}^{\infty} e_{j} x^{j} \in R[[x]]$.

From $e(x)^{2}=e(x)$, we have the following equations:

$$
\begin{aligned}
e_{0}^{2} & =e_{0} \\
e_{1} & =e_{0} e_{1}+e_{1} e_{0} \\
e_{2} & =e_{0} e_{2}+e_{1}^{2}+e_{2} e_{0} \\
& \vdots \\
e_{n} & =e_{0} e_{n}+e_{1} e_{n-1}+\cdots+e_{n-1} e_{1}+e_{n} e_{0}
\end{aligned}
$$

Multiplying Eq. (1) by $e_{0}$ on the left, we get $e_{0} e_{1}=e_{0} e_{1}+e_{0} e_{1} e_{0}$ and this gives $e_{0} e_{1} e_{0}=0$. Since $R$ is right IR, we have $e_{0} e_{1}=0$ and so this yields $e_{1}=e_{1} e_{0}$. 
Then from Eq. (2), we get $e_{2}=e_{0} e_{2}+e_{1}^{2}+e_{2} e_{0}=e_{0} e_{2}+e_{1} e_{0} e_{1}+e_{2} e_{0}=$ $e_{0} e_{2}+e_{2} e_{0}$ since $e_{0} e_{1}=0$. Multiplying this equation by $e_{0}$ on the left, we get $e_{0} e_{2}=e_{0} e_{2}+e_{0} e_{2} e_{0}$ and this gives $e_{0} e_{2} e_{0}=0$. Since $R$ is right IR, we have $e_{0} e_{2}=0$ and so this yields $e_{2}=e_{2} e_{0}$. We will proceed by induction. Assume that $e_{0} e_{j}=0, e_{j}=e_{j} e_{0}$ for $j \in\{1, \ldots, k-1\}$. Then

$$
\begin{aligned}
e_{k} & =e_{0} e_{k}+e_{1} e_{k-1}+\cdots+e_{k-1} e_{1}+e_{k} e_{0} \\
& =e_{0} e_{k}+e_{1} e_{0} e_{k-1}+\cdots+e_{k-1} e_{0} e_{1}+e_{k} e_{0} \\
& =e_{0} e_{k}+e_{k} e_{0} .
\end{aligned}
$$

Multiplying $e_{k}=e_{0} e_{k}+e_{k} e_{0}$ by $e_{0}$ on the left, we get $e_{0} e_{k}=e_{0} e_{k}+e_{0} e_{k} e_{0}$ and this gives $e_{0} e_{k} e_{0}=0$. Since $R$ is right IR, we have $e_{0} e_{k}=0$ and so this yields $e_{k}=e_{k} e_{0}$. Thus we now have

$$
e_{0}^{2}=e_{0} \text { and } e_{0} e_{j}=0, e_{j}=e_{j} e_{0} \text { for all } j \in\{1, \ldots, k\} .
$$

Thus the induction gives

$$
e_{0}^{2}=e_{0} \text { and } e_{0} e_{j}=0, e_{j}=e_{j} e_{0} \text { for all } j \in\{1,2, \ldots\} .
$$

Next from $f(x) e(x)=0$, we have the following equations:

$$
\begin{aligned}
0 & =a_{0} e_{0} \\
0 & =a_{0} e_{1}+a_{1} e_{0} \\
0 & =a_{0} e_{2}+a_{1} e_{1}+a_{2} e_{0} \\
& \vdots \\
0 & =a_{0} e_{n}+a_{1} e_{n-1}+\cdots+a_{n-1} e_{1}+a_{n} e_{0}
\end{aligned}
$$

$a_{0} e_{0}=0$ implies $e_{0} a_{0}=0$ since $R$ is right IR. Multiplying Eq. (4) by $e_{0}$ on the left, we get $0=e_{0} a_{0} e_{1}+e_{0} a_{1} e_{0}=e_{0} a_{1} e_{0}$ since $e_{0} a_{0}=0$. Since $R$ is right IR, we have $e_{0} a_{1}=0$. Multiplying Eq. (5) by $e_{0}$ on the left, we get $0=e_{0} a_{0} e_{2}+e_{0} a_{1} e_{1}+e_{0} a_{2} e_{0}=e_{0} a_{2} e_{0}$ since $e_{0} a_{0}=0$ and $e_{0} a_{1}=0$. Since $R$ is right IR, we have $e_{0} a_{2}=0$. We will proceed by induction. Assume that $e_{0} a_{i}=0$ for all $i \in\{0,1, \ldots, k-1\}$. Then

$$
\begin{aligned}
0 & =a_{0} e_{k}+a_{1} e_{k-1}+\cdots+a_{k-1} e_{1}+a_{k} e_{0} \\
& =e_{0} a_{0} e_{k}+e_{0} a_{1} e_{k-1}+\cdots+e_{0} a_{k-1} e_{1}+e_{0} a_{k} e_{0} \\
& =e_{0} a_{k} e_{0} .
\end{aligned}
$$

Since $R$ is right IR, we have $e_{0} a_{k}=0$. Thus we now have

$$
e_{0} a_{i}=0 \text { for all } i \in\{0,1, \ldots, k\} .
$$

Thus the induction gives

$$
e_{0} a_{i}=0 \text { for all } i \in\{0,1,2, \ldots\} .
$$


Now consider $e_{j} a_{i}$ for all $i, j$. If $j=0$, then $e_{j} a_{i}=0$ by the result (6). If $j \geq 1$, then $e_{j} a_{i}=e_{j} e_{0} a_{i}$ by the result (3) and so the result (6) implies $e_{j} a_{i}=e_{j} e_{0} a_{i}=0$. These yield $e(x) f(x)=0$ and thus $R[[x]]$ is right IR.

(2) comes from (1) and Lemma 2.13(2).

In the proof of Theorem 2.14, we obtain and use the fact that $e_{0} e(x)=e_{0}$ and $e(x) e_{0}=e(x)$.

The left IR condition also can go up to polynomial rings by similar arguments to the proof of the preceding theorem.

Corollary 2.15 ([14, Lemma 8]). Suppose that a ring $R$ is Abelian. Then we have the following results:

(1) Every idempotent of $R[x]$ is in $R$ and $R[x]$ is Abelian.

(2) Every idempotent of $R[[x]]$ is in $R$ and $R[[x]]$ is Abelian.

Proof. Let $R$ be Abelian. Then by the proof of Theorem 2.14, every idempotent in $R[[x]]$ is of the form $e^{2}=e \in R$. The rest is obtained from Theorem 2.14 and Corollary 2.9.

Lastly we study the minimality for non-Abelian right (left) IIP (IR) rings. $\left(\begin{array}{ll}0 & \mathbb{Z}_{2} \\ 0 & \mathbb{Z}_{2}\end{array}\right)$ (resp. $\left.\left(\begin{array}{cc}\mathbb{Z}_{2} & \mathbb{Z}_{2} \\ 0 & 0\end{array}\right)\right)$ is right (resp. left) IIP by Example 2.3(4) (resp. Example 2.3(3)), but not left (resp. right) IR as can be seen by $e_{22} e_{12}=0 \neq e_{12}=$ $e_{12} e_{22}$ (resp. $\left.e_{11} e_{12}=e_{12} \neq 0=e_{12} e_{11}\right)$. Given a ring $R$, the upper nilradical (i.e., the sum of all nil ideals) and the set of all nilpotent elements in $R$ are denoted by $N^{*}(R)$ and $N(R)$, respectively.

Theorem 2.16. For a ring $R$, we have the following results:

(1) If $R$ is a minimal non-Abelian right IIP ring, then $R$ is isomorphic to $\left(\begin{array}{ll}0 & \mathbb{Z}_{2} \\ 0 & \mathbb{Z}_{2}\end{array}\right)$.

(2) If $R$ is a minimal non-Abelian left IIP ring, then $R$ is isomorphic to $\left(\begin{array}{cc}\mathbb{Z}_{2} & \mathbb{Z}_{2} \\ 0 & 0\end{array}\right)$

(3) If $R$ is a minimal non-Abelian right $I R$ ring, then $R$ is isomorphic to $\left(\begin{array}{ll}0 & \mathbb{Z}_{2} \\ 0 & \mathbb{Z}_{2}\end{array}\right)$.

(4) If $R$ is a minimal non-Abelian left IR ring, then $R$ is isomorphic to $\left(\begin{array}{cc}\mathbb{Z}_{2} & \mathbb{Z}_{2} \\ 0 & 0\end{array}\right)$.

Proof. Let $R$ be a minimal non-Abelian right IR ring. Then $|R|=4$ by the existence of the right IR ring $\left(\begin{array}{ll}0 & \mathbb{Z}_{2} \\ 0 & \mathbb{Z}_{2}\end{array}\right)$.

If $R$ is nilpotent, then by [15, Theorem 2.3.3], there is a basis $\{a, b\}$ for $R$ over $\mathbb{Z}_{2}$ such that $a^{2}=b^{2}=a b=b a=0$ or $a^{2}=b, a^{3}=0$. This implies that $R$ is commutative, a contradiction.

Suppose $J(R)=0$. Here assume that the characteristic of $R$ is $\geq 3$. Then there exists a nonzero $x \in R$ with $|x| \geq 3$. This yields $|x|=4$ since $|R|=4$, entailing $R=\{0, x, 2 x, 3 x\}$. So $R$ is commutative, a contradiction. Thus the characteristic of $R$ is 2 . Whence we can construct an extension ring $R^{1}$ by attaching an identity to $R$, i.e., $R^{1}=R \times \mathbb{Z}_{2}$ with $\left(x_{1}, n_{1}\right)+\left(x_{2}, n_{2}\right)=$ 
$\left(x_{1}+x_{2}, n_{1}+n_{2}\right)$ and $\left(x_{1}, n_{1}\right)\left(x_{2}, n_{2}\right)=\left(x_{1} x_{2}+n_{1} x_{2}+n_{2} x_{1}, n_{1} n_{2}\right)$. Then $R^{1}$ has the identity $(0,1)$ and a subring $R \times 0 \cong R$. Moreover since $J(R)=0$, we also have $J\left(R^{1}\right)=0$. By the Wedderburn-Artin theorem, $R^{1} \cong \operatorname{Mat}_{n_{1}}\left(\mathbb{Z}_{2}\right) \oplus$ $\cdots \oplus \operatorname{Mat}_{n_{k}}\left(\mathbb{Z}_{2}\right)$ for some $n_{i} \geq 1(i=1, \ldots, k)$. But $\left|R^{1}\right|=8$, and so every $n_{i}$ must be 1 , yielding $k=3$. Then $R^{1}$ is commutative and so is $R$, a contradiction.

We now have that $R$ has non-nilpotent and $J(R) \neq 0$. This yields $|J(R)|=2$, and this implies $R / J(R) \cong \mathbb{Z}_{2}$. By [16, Proposition 3.6.2], there exists an idempotent $e \in R$ such that $1+J(R)=e+J(R)$. Since $R$ is non-Abelian, there exists $b \in R$ such that $e b \neq b e$, entailing $R=\{0, e, b, e+b\}$ and $J(R)=\{0, b\}$, $b^{2}=0$. Let $e b \neq 0$ and assume $e b=e+b$. Then $0=e b b=e b+b b=e b$ and so $e b=b$ (if $e b=e$, then $0=e b b=e b$ ). Similarly $b e=b$ when $b e \neq 0$. Since $b e \neq e b$, we have two cases

$$
(b e=b, e b=0) \text { and }(e b=b, b e=0) .
$$

If $R$ is right IR, then $R$ is the former case; and if $R$ is left IR, then $R$ is the latter case. Next let $R_{1}=\left(\begin{array}{ll}0 & \mathbb{Z}_{2} \\ 0 & \mathbb{Z}_{2}\end{array}\right)$ and $R_{2}=\left(\begin{array}{cc}\mathbb{Z}_{2} & \mathbb{Z}_{2} \\ 0 & 0\end{array}\right)$. In the former case, $R \cong R_{1}$ by $e \mapsto e_{22}, b \mapsto e_{12}$; and in the latter case, $R \cong R_{2}$ by $e \mapsto e_{11}, b \mapsto e_{12}$. These complete the proof since right (resp. left) IIP ring is right (resp. left) IR by Lemma 2.4.

\section{Abelian rings of minimal order}

In this section we observe the classes of minimal Abelian rings. The following lemma is a base for our process.

Lemma 3.1. Let $R$ be an Abelian ring with identity. Then $R$ is semiperfect if and only if $R$ is a finite direct sum of local rings.

Proof. Suppose that $R$ is semiperfect. Then $R$ has a finite orthogonal set of local idempotents whose sum is 1 by [16, Proposition 3.7.2], say $R=\sum_{i=1}^{n} e_{i} R$ such that each $e_{i} R e_{i}$ is a local ring. Since $R$ is Abelian, each $e_{i} R$ is an ideal of $R$ with $e_{i} R=e_{i} R e_{i}$. The converse is trivial.

First note the following kinds of Abelian rings. A ring is called right (resp. left) duo if every right (resp. left) ideal is two-sided, due to Feller [10]. It is obvious that one-sided duo rings are IFP.

Example 3.2. (1) $R_{1}=D_{3}\left(\mathbb{Z}_{2}\right)$ is IFP by [14, Proposition 1.2]. So $R_{1}$ is a noncommutative Abelian ring of order 16 over $\mathbb{Z}_{2}$. Note that $R_{1}$ is neither left nor right duo. Next consider $A=\mathbb{Z}_{2}\langle x, y\rangle$, the free algebra with noncommuting indeterminates $x, y$ over the field $\mathbb{Z}_{2}$. Due to $\mathrm{Xu}$ and Xue [22, Example 7], let $I$ be the ideal of $A$ generated by

$$
x^{3}, y^{2}, y x, x^{2}-x y
$$

and $B=A / I$. Then $B$ is IFP by the argument in [22, Example 7]. But $R_{1}$ is isomorphic to $B$ through the correspondence $x \mapsto e_{12}+e_{23}$ and $y \mapsto e_{23}$. 
(2) The construction is due to Xue [21, Example 2]. Note that

$$
R_{2}=\left\{\left(\begin{array}{cc}
a & b \\
0 & a^{2}
\end{array}\right) \mid a, b \in G F\left(2^{2}\right)\right\}
$$

is duo (hence IFP) by the computation in [21, Example 2]. So $R_{2}$ is a noncommutative Abelian ring of order 16 over $G F\left(2^{2}\right)$.

(3) The construction is due to Xue [21, Example 2]. Let $\mathbb{Z}_{4}\langle x, y\rangle$ be the free algebra with noncommuting indeterminates $x, y$ over $\mathbb{Z}_{4}$. Let $R_{3}=\mathbb{Z}_{4}\langle x, y\rangle / I$ where $I$ is the ideal of $\mathbb{Z}_{4}\langle x, y\rangle$ generated by $x^{3}, y^{3}, y x, x^{2}-x y, x^{2}-2, y^{2}-$ $2,2 x, 2 y$. Then $R_{3}$ is duo (hence IFP) by the computation in [21, Example 2]. So $R_{3}$ is a noncommutative Abelian ring of order 16 over $\mathbb{Z}_{4}$.

(4) The construction is due to Xue [21, Example 2]. Let $R=\mathbb{Z}_{2}\langle x, y\rangle$ be the free algebra with noncommuting indeterminates $x, y$ over the field $\mathbb{Z}_{2}$. Let $I$ be the ideal of $R$ generated by

$$
x^{3}, y^{3}, y x, x^{2}-x y, y^{2}-x y
$$

and $R_{4}=R / I$. Then $R_{4}$ is duo (hence IFP) by the computation in [21, Example 2]. So $R_{4}$ is a noncommutative Abelian ring of order 16 over $\mathbb{Z}_{2}$.

(5) The construction is due to $\mathrm{Xu}$ and Xue [22, Example 7]. Let $R=\mathbb{Z}_{4}\langle x, y\rangle$ be the free algebra with noncommuting indeterminates $x, y$ over $\mathbb{Z}_{4}$. Let $I$ be the ideal of $R$ generated by

$$
x^{3}, y^{2}, y x, x^{2}-x y, x^{2}-2,2 x, 2 y
$$

and $R_{5}=R / I$. Then $R_{5}$ is IFP by the argument in [22, Example 7]. So $R_{5}$ is a noncommutative Abelian ring of order 16 over $\mathbb{Z}_{4}$.

The rings in Example 3.2 are minimal noncommutative Abelian rings with identity as we see in the following theorem.

Theorem 3.3. Let $R$ be a ring with identity. If $R$ is a minimal noncommutative Abelian ring, then $R$ is of order 16 and is isomorphic to $R_{i}$ for some $i \in\{1,2,3,4,5\}$, where $R_{i}$ 's are the rings in Example 3.2.

Proof. We apply the proof of [11, Theorem 2.6]. Eldridge proved that a finite ring is commutative when its order has a cube free factorization in $[8$, Theorem], and that if a finite noncommutative ring is of order $p^{3}, p$ a prime, then it is isomorphic to $U_{2}(G F(p))$ in [8, Proposition]. Thus every minimal noncommutative ring is isomorphic to $U_{2}\left(\mathbb{Z}_{2}\right)$. But $U_{2}\left(\mathbb{Z}_{2}\right)$ is non-Abelian, and so a minimal noncommutative Abelian ring is of order 16 by the ring in Example $3.2(1)$.

Let $R$ be a minimal noncommutative Abelian ring. Then $R$ is a local ring by Lemma 3.1 since $R$ is minimal. Here we have three cases: $|J(R)|=2$, $|J(R)|=4$ or $|J(R)|=8$, where $|J(R)|$ means the cardinality of $J(R)$. Note that $R / J(R)$ is a field and $J(R)$ is a vector space over $R / J(R)$.

The case of $|J(R)|=2$ is impossible by the proof of [11, Theorem 2.6]. 
Consider the case of $|J(R)|=4$. In this case $R$ is isomorphic to

$$
\left\{\left(\begin{array}{cc}
a & b \\
0 & a^{2}
\end{array}\right) \mid a, b \in G F\left(2^{2}\right)\right\}
$$

by the proof of $[11$, Theorem 2.6].

Lastly suppose $|J(R)|=8$. Then $R / J(R) \cong \mathbb{Z}_{2}$, and $J(R)$ is a nilpotent algebra of dimension 3 over $\mathbb{Z}_{2}$. Thus, by Kruse and Price [15, Theorem 2.3.6], $J(R)$ has a basis $\{a, b, c\}$, with $J(R) c=c J(R)=0$, such that one of the following conditions holds:

(1) $a^{2}=a b=b a=b^{2}=0$;

(2) $a^{2}=b^{2}=0, a b=-b a=c$;

(3) $a^{2}=c, a b=b a=b^{2}=0$;

(4) $a^{2}=c, a b=b a=0, b^{2}=c$;

(5) $a^{2}=a b=c, b a=0, b^{2}=\phi c$ for some $\phi \in \mathbb{Z}_{2}$ (i.e., $b^{2}=0$ or $\left.b^{2}=c\right)$;

(6) $a^{2}=b, a^{3}=c, a^{4}=0$, where all of the preceding algebras are mutually non-isomorphic.

Since $R=\{a, 1+a \mid a \in J(R)\}$, we have that $R$ is commutative if and only if so is $J(R)$. Consider the case (2). Since $J(R)$ is a vector space over $\mathbb{Z}_{2}$, $v=-v$ for all $v \in J(R)$, entailing $a b=-b a=b a$. In the case (6), $a b=b a$ clearly. Thus $R$ is commutative when $J(R)$ satisfies one of the conditions (1), $(2),(3),(4)$, and (6). So it suffices to examine the condition (5) to complete the proof.

Consider the ring $R_{1}$ in Example 3.2(1). Recall $J\left(R_{1}\right)=\left(\begin{array}{ccc}0 & \mathbb{Z}_{2} & \mathbb{Z}_{2} \\ 0 & 0 & \mathbb{Z}_{2} \\ 0 & 0 & 0\end{array}\right)$. Let $a=e_{12}+e_{23}, b=e_{23}, c=e_{13} \in J\left(R_{1}\right)$. Then $J\left(R_{1}\right)$ satisfies the condition (5) with $J\left(R_{1}\right) c=c J\left(R_{1}\right)=0$ and $b^{2}=0=0 c$.

Consider the ring $R_{3}$ in Example $3.2(3)$ and recall $J\left(R_{3}\right)=2 \mathbb{Z}_{4}+R_{2} x R_{2}+$ $R_{2} y R_{2}$. Let $a=\bar{x}-\bar{y}, b=\bar{x}, c=\overline{2} \in J\left(R_{3}\right)$. Then $J\left(R_{3}\right)$ satisfies the condition (5) with $J\left(R_{3}\right) c=c J\left(R_{3}\right)=0$ and $b^{2}=c=1 c$.

Consider the ring $R_{4}$ in Example 3.2(4) and recall $J\left(R_{4}\right)=(\bar{x}, \bar{y})$. Let $a=\bar{x}, b=\bar{y}, c=\bar{x}^{2} \in J\left(R_{4}\right)$. Then $J\left(R_{4}\right)$ satisfies the condition (5) with $J\left(R_{4}\right) c=c J\left(R_{4}\right)=0$ and $b^{2}=c=1 c$.

Consider the ring $R_{5}$ in Example 3.2(5) and recall $J\left(R_{5}\right)=(\bar{x}, \bar{y}, \overline{2})$. Let $a=\bar{x}, b=\bar{y}, c=\overline{2} \in J\left(R_{5}\right)$. Then $J\left(R_{5}\right)$ satisfies the condition (5) with $J\left(R_{5}\right) c=c J\left(R_{5}\right)=0$ and $b^{2}=0=0 c$.

Note that $R_{i}$ is not isomorphic to $R_{j}$ for $i \neq j$ (where $i, j \in\{1,2,3,4,5\}$ ) by the arguments in [22, Example 7] and [21, Example 2].

In the following argument, the minimal noncommutative Abelian rings without identity is completely determined, up to isomorphism. This result also yields that a ring is a minimal noncommutative Abelian ring if and only if it is a minimal noncommutative nilpotent ring. Therefore this result leads to methods by which noncommutative Abelian or nil rings are constructed. 
Example 3.4. (1) $E=\left\{\left(a_{i j}\right) \in U_{n}(A) \mid a_{i j}=0\right.$ when $\left.i=j\right\}$ is a nilpotent ring for any ring $A$. $E$ is commutative when $n=1,2$. While, $E$ is noncommutative when $n \geq 3$ and $A$ has an identity (as can be seen by $e_{12} e_{23}=e_{13}$ and $\left.e_{23} e_{12}=0\right)$.

(2) According to Xue [21, Example 2], let $T=\mathbb{Z}_{4}\langle x, y\rangle / I$ where $\mathbb{Z}_{4}\langle x, y\rangle$ is the free algebra with noncommuting indeterminates $x, y$ over $\mathbb{Z}_{4}$ and $I$ is the ideal of $\mathbb{Z}_{4}\langle x, y\rangle$ generated by $x^{3}, y^{3}, y x, x^{2}-x y, x^{2}-2, y^{2}-2,2 x, 2 y$. Then $J(T)=(2, x, y)$ is noncommutative, and since $a b c=0$ for $a, b, c \in\{2, x, y\}$, we get $J(T)^{3}=0$.

(3) Let $\mathbb{Z}_{4}\langle x, y, z\rangle$ be the free algebra with noncommuting indeterminates $x, y, z$ over $\mathbb{Z}_{4}$. Construct the following factor rings:

$$
\begin{aligned}
& E_{1}=\mathbb{Z}_{4}\langle x, y, z\rangle /\left(x^{2}-z, y^{2}-z, x y-z, 2 x-z, 2 y-z, y x, 2 z, x z, y z, z x, z y, z^{2}\right) ; \\
& E_{2}=\mathbb{Z}_{4}\langle x, y, z\rangle /\left(x^{2}-z, y^{2}-z, x y-z, 2 x-z, y x, 2 y, 2 z, x z, y z, z x, z y, z^{2}\right) ; \\
& E_{3}=\mathbb{Z}_{4}\langle x, y, z\rangle /\left(x^{2}-z, y^{2}, x y-z, 2 x-z, 2 y-z, y x, 2 z, x z, y z, z x, z y, z^{2}\right) ; \\
& E_{4}=\mathbb{Z}_{4}\langle x, y, z\rangle /\left(x^{2}-z, y^{2}, x y-z, 2 x-z, y x, 2 y, 2 z, x z, y z, z x, z y, z^{2}\right) .
\end{aligned}
$$

Next define

$$
R_{i}=\left\{f \in E_{i} \mid f \text { has a zero constant term }\right\}
$$

for all $i$. Identify each element in $\mathbb{Z}_{4}\langle x, y, z\rangle$ and its image in $E_{i}$ for simplicity. Note that $z^{2}=0, x^{4}=0$ ( since $\left.x^{4}=z^{2}=0\right), 2 z=0, x^{2}=x y=2 x=z$, and $x z=y z=z x=z y=y x=0$ in any $R_{i}$. Since $x^{2}-z=0, x^{3}=x^{2} x=z x=0$, and similarly $y^{3}=0$. Note that $R_{i}=\langle x, y, z\rangle=\mathbb{Z}_{4} x \oplus \mathbb{Z}_{4} y \oplus \mathbb{Z}_{4} z=\{0, x, y, x+$ $y, z, z+x, z+y, z+x+y\}$. Note also that every $R_{i}$ is a noncommutative ring. $y^{2}=z \neq 0$ in $R_{i}$ for $i=1,2$, and $y^{2}=0$ in $R_{i}$ for $i=3,4 ;|x|=|y|=4,|z|=2$ in $R_{i}$ for $i=1,3$, and $|x|=4,|y|=|z|=2$ in $R_{i}$ for $i=2,4$. These imply that $R_{i}$ 's are mutually non-isomorphic.

$R_{i}^{2}=\{0, z\}$ for all $i$ and $\left(R_{i} / R_{i}^{2}\right)^{+} \cong \mathbb{Z}_{2} \oplus \mathbb{Z}_{2}$, where $\left\{0, x+R_{i}^{2}\right\} \cong \mathbb{Z}_{2}$ and $\left\{0, y+R_{i}^{2}\right\} \cong \mathbb{Z}_{2}$. In any $R_{i}, a b c=(a b) c=z c=0$ (when $\left.a b \neq 0\right)$ for all $a, b, c \in\{x, y, z\}$; hence $R_{i}^{3}=0$. Note $\operatorname{char} R=4$.

(4) Both $\left(\begin{array}{ll}A & A \\ 0 & 0\end{array}\right)$ and $\left(\begin{array}{ll}0 & A \\ 0 & A\end{array}\right)$ are non-Abelian rings for any ring $A$ with identity.

In Theorem 3.3, $R$ is a minimal noncommutative Abelian ring with identity, then $R$ is of order 16 and is isomorphic to $D_{3}\left(\mathbb{Z}_{2}\right)$, the second ring in $[21$, Example 2], or the ring of all matrices of the form $\left(\begin{array}{cc}a & b \\ 0 & a^{2}\end{array}\right)$ over $G F\left(2^{2}\right)$. We will observe the class of minimal noncommutative Abelian rings without identity. The following lemma was shown by Erickson [9] and Eldridge [8]. In [5], Bell called a ring $R$ periodic if for each $x \in R$, there exist distinct positive integers $n, m$, depending on $x$, for which $x^{n}=x^{m}$. Finite rings are clearly periodic.

Lemma 3.5. (1) [9, Theorem 1] If $R$ is a ring of order $n>1$ and if $n$ has square free factorization, then $R$ is a commutative ring.

(2) [9, Theorem 2] If $p$ is a prime integer, then there exists a noncommutative ring of order $p^{2}$. 
(3) $[8$, Theorem] Let $R$ be a finite ring of order $m$ with identity. If $m$ has a cube free factorization, then $R$ is a commutative ring.

(4) [5, Lemma 1(a)] If $R$ is a periodic ring, then some power of $x$ is an idempotent for each $x \in R$.

(5) [2, Proposition 27.1] If $I$ is a nil ideal in a ring $R$, then idempotents lift modulo $I$.

One can say that any finite ring is a commutative ring when its order has a square free factorization, by Lemma $3.5(1),(3)$.

Theorem 3.6. If $R$ is a minimal noncommutative Abelian ring, then $R$ is of order 8 and is isomorphic to $\left(\begin{array}{ccc}0 & \mathbb{Z}_{2} & \mathbb{Z}_{2} \\ 0 & 0 & \mathbb{Z}_{2} \\ 0 & 0 & 0\end{array}\right), J(T)$ in Example $3.4(2)$, or one of $R_{i}$ 's $(i=1,2,3,4)$ in Example 3.4(3).

Proof. Let $R$ be a minimal noncommutative Abelian ring. Erickson decided that the order of a finite noncommutative ring must have a square factor, by Lemma 3.5(1), (2). Furthermore Eldridge claimed that such a ring does not have an identity, by Lemma 3.5(3). If $R^{+}$is cyclic, then $R$ is commutative and so $R^{+}$is not cyclic.

Assume $|R|=4$, based on Lemma 3.5(2). If $R$ is nilpotent (i.e., $J(R)=R$ ), then there exists a basis $\{a, b\}$ for $R$ over $\mathbb{Z}_{2}$ such that $a^{2}=b^{2}=a b=b a=0$ or $a^{2}=b, a^{3}=0$, by [15, Theorem 2.3.3]. This implies that $R$ is commutative, a contradiction. If $J(R)=0$, then $R$ is commutative by the proof of Theorem 2.16. Consequently $R$ is a non-nilpotent non-semiprimitive ring. Then we must have $|J(R)|=2$ and $R / J(R) \cong \mathbb{Z}_{2}$. By Lemma 3.5(5), there exists an idempotent $e \in R$ such that $a+J(R)=e+J(R)$ where $a+J(R)$ is the identity of $R / J(R)$. Consequently we get $R=\{0, e, b, e+b\}$, letting $J(R)=\{0, b\}$. But since $R$ is Abelian, $e b=b e$ and so $R$ is commutative, a contradiction.

Therefore the order of $R$ must be larger than 4 . Then $|R| \geq 8$ by Lemma $3.5(1)$, but the existence of the Abelian ring $\left(\begin{array}{ccc}0 & \mathbb{Z}_{2} & \mathbb{Z}_{2} \\ 0 & 0 & \mathbb{Z}_{2} \\ 0 & 0 & 0\end{array}\right)$ concludes $|R|=8$. Thus we can conclude that a minimal noncommutative Abelian ring must be of order 8. Since $J(R)$ is an additive subgroup of $R, J(R)$ is of order 2,4 , or 8 .

Suppose $|J(R)|=4$. Then the characteristic of $J(R)$ is either 2 or 4 . Assume $\operatorname{char} J(R)=4$. Then there exists a nonzero $x \in R$ with $\operatorname{char} x \geq 3$. This yields $\operatorname{char} x=4$ since $|J(R)|=4$, entailing $J(R)=\{0, x, 2 x, 3 x\}$. Assume char $J(R)=2$. Then, by [15, Theorem 2.3.3], there exists a basis $\{a, b\}$ for $J(R)$ over $\mathbb{Z}_{2}$ such that $a^{2}=b^{2}=a b=b a=0$ or $a^{2}=b, a^{3}=0$. In any case $J(R)$ is a commutative ring. Note $(R / J(R))^{+} \cong \mathbb{Z}_{2}$, say $R / J(R)=$ $\{0, a+J(R)\}$. Since $R / J(R)$ is not nil, $a+J(R)$ must be an identity of $R / J(R)$. By Lemma 3.5(5), there exists a nonzero idempotent $e \in R$ such that $a+J(R)=e+J(R)$. Consequently we get $R=\{j, e+j \mid j \in J(R)\}$. But since $R$ is Abelian, $e x=x e$ and $e a=a e, e b=b e$. Then $R$ is commutative since $J(R)$ is commutative. This is a contradiction. Thus $|J(R)| \neq 4$.

Suppose $|J(R)|=2$ and $J(R)=\{0, a\}$. Then $(R / J(R))^{+}$is isomorphic to either $\mathbb{Z}_{4}$ or $\mathbb{Z}_{2} \oplus \mathbb{Z}_{2}$ by the Fundamental Theorem of Finite Abelian Groups. 
Assume $(R / J(R))^{+} \cong \mathbb{Z}_{4}$ and say $R / J(R)=\{0, x+J(R), 2 x+J(R), 3 x+$ $J(R)\}$. Then $(2 x+J(R))^{2}=0$ in $R / J(R)$, and so the ideal of $R$ generated by $\{2 x\} \cup J(R)$ is nil. This yields $2 x \in J(R)$, a contradiction. Assume $(R / J(R))^{+} \cong \mathbb{Z}_{2} \oplus \mathbb{Z}_{2}$. Since $R / J(R)$ is not nil, $z+J(R)$ is non-nilpotent for some $z+J(R) \in R / J(R)$. Then some power of $z+J(R)$ is a nonzero idempotent in $R / J(R)$ by Lemma 3.5(4), say $z^{m}+J(R)$. By Lemma 3.5(5), there exists a nonzero idempotent $e \in R$ such that $z^{m}+J(R)=e+J(R)$. Consider the ideal $K$ of $R$ generated by $\{e\} \cup J(R)$. If $K=R$, then $R$ is commutative since $R$ is Abelian. This induces a contradiction, and so $K \varsubsetneqq R$. Note $R / K \cong \mathbb{Z}_{2}$ and say $R / K=\{0, x+K\}$. Since the characteristic of $R / J(R)$ is $2,2 x \in J(R)$ and $(R / J(R))^{+} \cong\{0, e\} \oplus\{0, x\}$, entailing $R=\{0, e, x, e+x, a, a+e, a+x, a+e+x\}$. If $x+J(R)$ is nilpotent, then $x$ is nilpotent. Moreover since $e$ is central, the subset $\{x\} \cup J(R)$ generates a nil ideal of $R$. This leads to $x \in J(R)$, a contradiction. Thus $x+J(R)$ is non-nilpotent in $J(R)$; hence by Lemma 3.5(4,5), there exists a nonzero idempotent $f \in R$ such that $x^{n}+J(R)=f+J(R)$ for some $n \geq 1$. Here let $n$ be the smallest such integer.

Assume $e=f$. Then $e+J(R)=x^{n}+J(R)$ and so $e=x^{n}$ or $e=x^{n}+a$. Moreover $R / J(R)=\left\{0, x+J(R), x^{2}+J(R), x^{3}+J(R)\right\}$ and $x^{4}+J(R) \in$ $\left\{x+J(R), x^{2}+J(R), x^{3}+J(R)\right\}$, since $|R / J(R)|=4$ and $x+J(R)$ is nonnilpotent. We will show $x a=a x$. Then $R$ is commutative since every element of $R$ is of the form $x^{s}$ or $x^{s}+a$. Assume on the contrary that $x a \neq a x$. Say $x a=a, a x=0$. Then $x^{k} a=a$ for all $k \geq 1$. So if $e=x^{n}+a$, then

$$
\begin{aligned}
0 & =0+0=a x^{n}+a^{2}=a\left(x^{n}+a\right)=a e=e a \\
& =\left(x^{n}+a\right) a=x^{n} a+a^{2}=a+0=a \neq 0,
\end{aligned}
$$

a contradiction. Thus $e=x^{n}$, and so we also get

$$
0 \neq a=x^{n} a=e a=a e=a x^{n}=0,
$$

a contradiction. The case of $x a=0, a x=a$ also induces a contradiction by a similar method. Consequently $a x=x a$, a contradiction. Therefore $e \neq f$, and this yields $R=\{0, e, f, e+f, a, a+e, a+f, a+e+f\}$. Whence $R$ is commutative since $R$ is Abelian, a contradiction. Thus $|J(R)| \neq 2$.

These conclude that it suffices to argue only about the case when $|J(R)|=8$. Suppose $J(R)=R$. Then $R$ is nilpotent, and $|R|>\left|R^{n}\right|$ for any $n \geq 2$. If $R^{2}=0$, then $R$ is commutative and so $R^{2} \neq 0$, entailing that $\left|R^{2}\right|=2$ or $\left|R^{2}\right|=4$. If $\left|R^{2}\right|=4$, then $\left|R / R^{2}\right|=2$ and so there exists a generator $a+R^{2}$ for $\left(R / R^{2}\right)^{+}$. Then $R$ is the ring generated by a by [15, Lemma 2.3.1], and so $R$ is commutative. This is a contradiction, and so $\left|R^{2}\right|=2$. If $\left(R / R^{2}\right)^{+}$ is cyclic, then $R$ is also commutative by [15, Lemma 2.3.1]. Thus $\left(R / R^{2}\right)^{+}$ is not cyclic, leading to $\left(R / R^{2}\right)^{+} \cong \mathbb{Z}_{2} \oplus \mathbb{Z}_{2}$. Note $\left(R^{2}\right)^{+} \cong \mathbb{Z}_{2}$, and say $R^{2}=\{0, c\}$. Clearly $2 c=0$ and $c^{2}=0$. Since $\left(R / R^{2}\right)^{+} \cong \mathbb{Z}_{2} \oplus \mathbb{Z}_{2}$, there exist $a, b \in R$ such that $\left(R / R^{2}\right)^{+}=\left\{0, a+R^{2}, b+R^{2}, a+b+R^{2}\right\}$ and $2 a \in$ $R^{2}, 2 b \in R^{2}$. Then $R=\{0, a, b, a+b, c, a+c, b+c, a+b+c\}$. Here assume $|a|=4$. Then $2 a=c$ and $a c=c a$. If $a^{2} \neq 0$, then $a^{2}=c$ since $a^{2} \in R^{2}$. 
Whence $c a=(2 a) a=2 a^{2}=2 c=0$ and this yields that $a^{3}=a^{2} a=c a=0$ when $a^{2}$ is zero or not. A similar calculation gives $b^{3}=0$ and $b c=c b$ when $|b|=4$. Since $R$ is noncommutative, we must have $a b \neq b a$ and so either $a b=c, b a=0$ or $a b=0, b a=c$. Consequently, if $|R|=8$, then $R^{3}=0$ since $x y z=(x y) z=(2 a) z=2 c=0$ or $x y z=(x y) z=(2 b) z=2 c=0$ (when $x y, a z, b z$ are all nonzero) for every $x, y, z \in\{a, b, c\}$. Thus we have the following four cases:

$$
\begin{aligned}
& |a|=|b|=4,|c|=2, a^{2}=b^{2}=a b=2 a=2 b=c, \\
& b a=a c=c a=b c=c b=c^{2}=0 \\
& |a|=4,|b|=|c|=2, a^{2}=b^{2}=a b=2 a=c, \\
& b a=a c=c a=b c=c b=c^{2}=0 \\
& |a|=|b|=4,|c|=2, a^{2}=a b=2 a=2 b=c, \\
& b^{2}=b a=a c=c a=b c=c b=c^{2}=0 \\
& |a|=4,|b|=|c|=2, a^{2}=a b=2 a=c \\
& b^{2}=b a=a c=c a=b c=c b=c^{2}=0 .
\end{aligned}
$$

Thus $R$ is isomorphic to $R_{1}$ in Example 3.4(3) for the case (1), $R$ is isomorphic to $R_{2}$ in Example $3.4(3)$ for the case (2), $R$ is isomorphic to $R_{3}$ in Example $3.4(3)$ for the case (3), and $R$ is isomorphic to $R_{4}$ in Example 3.4(3) for the case (4), through the correspondence $a \mapsto x, b \mapsto y, c \mapsto z$.

Next assume that $R$ is an algebra of dimension 3 over $\mathbb{Z}_{2}$. Then, by Kruse and Price [15, Theorem 2.3.6], $J(R)$ has a basis $\{a, b, c\}$, with $R c=c R=0$, that one of the following conditions holds:

$$
\begin{aligned}
& a^{2}=a b=b a=b^{2}=0 ; \\
& a^{2}=b^{2}=0, a b=-b a=c ; \\
& a^{2}=c, a b=b a=b^{2}=0 ; \\
& a^{2}=c, a b=b a=0, b^{2}=c ; \\
& a^{2}=a b=c, b a=0, b^{2}=\phi c \text { for some } \phi \in \mathbb{Z}_{2} ; \\
& a^{2}=b, a^{3}=c, a^{4}=0,
\end{aligned}
$$

where all of the preceding algebras are mutually non-isomorphic. However $R$ is commutative when $R$ satisfies one of the conditions (5), (6), (7), (8), and (10). So it suffices to concentrate on the condition (9). We next apply the proof of Theorem 3.3.

Consider the ring $R_{1}=\left(\begin{array}{ccc}0 & \mathbb{Z}_{2} & \mathbb{Z}_{2} \\ 0 & 0 & \mathbb{Z}_{2} \\ 0 & 0 & 0\end{array}\right)$ and let $a=e_{12}+e_{23}, b=e_{23}, c=e_{13} \in R_{1}$. Then $R_{1}$ satisfies the condition (9) with $R_{1} c=c R_{1}=0$ and $b^{2}=0=0 c$.

Next set $R_{2}=J(T)$ in Example $3.4(2)$ and let $a=\bar{x}-\bar{y}, b=\bar{x}, c=\overline{2}$. Then $R_{2}$ satisfies the condition (9) with $R_{2} c=c R_{2}=0$ and $b^{2}=c$. 
Compare $R_{1}$ and $R_{2}$. In $R_{1}, f=e_{12}+e_{23}$ and $g=e_{12}+e_{13}+e_{23}$ are all elements such that $f^{2}=c=g^{2}$ and they are of index 3 of nilpotency. But $f g=g f=e_{13} \neq 0$. Thus $R_{1}$ is not isomorphic to $R_{2}$, noting that $b^{2}=0 c$ in $R_{1}$ and $b^{2}=1 c$ in $R_{2}$.

By the proof of Theorem 3.6, we can obtain the following corollary.

Corollary 3.7. If $R$ is a minimal noncommutative nilpotent ring, then $R$ is of order 8 and is isomorphic to $\left(\begin{array}{ccc}0 & \mathbb{Z}_{2} & \mathbb{Z}_{2} \\ 0 & 0 & \mathbb{Z}_{2} \\ 0 & 0 & 0\end{array}\right), J(T)$ in Example 3.4(2), or one of $R_{i}$ 's $(i=1,2,3,4)$ in Example $3.4(3)$.

We get the following result by help of Theorem 3.6 and Corollary 3.7.

Corollary 3.8. A ring $R$ is minimal noncommutative Abelian if and only if $R$ is a minimal noncommutative nilpotent ring.

A quick computation gives that a ring $R$ is a minimal (commutative) nilpotent ring then $R$ is isomorphic to $\left(\begin{array}{cc}0 & \mathbb{Z}_{2} \\ 0 & 0\end{array}\right)$. Note that $2 \mathbb{Z}_{4} \cong\left(\begin{array}{ll}0 & \mathbb{Z}_{2} \\ 0 & 0\end{array}\right)$.

Acknowledgements. The authors thank the referee for very careful reading of the manuscript and many valuable suggestions that improved the paper by much. This work was supported by a 2-Year Research Grant of Pusan National University.

\section{References}

[1] D. D. Anderson and V. Camillo, Semigroups and rings whose zero products commute, Comm. Algebra 27 (1999), no. 6, 2847-2852.

[2] F. W. Anderson and K. R. Fuller, Rings and Categories of Modules, Springer-Verlag, New York, 1992.

[3] G. Azumaya, Strongly $\pi$-regular rings, J. Fac. Sci. Hokkaido Univ. Ser. I. 13 (1954), 34-39.

[4] H. E. Bell, Near-rings in which each element is a power of itself, Bull. Austral. Math. Soc. 2 (1970), 363-368.

[5] $\longrightarrow$, A commutativity study for periodic rings, Pacific J. Math. 70 (1977), no. 1, $29-36$.

[6] P. M. Cohn, Reversible rings, Bull. London Math. Soc. 31 (1999), no. 6, 641-648.

[7] F. Dischinger, Sur les anneaux fortement $\pi$-réguliers, C. R. Acad. Sci. Paris Sér. A-B 283 (1976), no. 8, 571-573.

[8] K. E. Eldridge, Orders for finite noncommutative rings with unity, Amer. Math. Monthly 75 (1968), no. 5, 512-514.

[9] D. B. Erickson, Orders for finite noncommutative rings, Amer. Math. Monthly 73 (1966), 376-377.

[10] E. H. Feller, Properties of primary noncommutative rings, Trans. Amer. Math. Soc. 89 (1958), 79-91.

[11] C. Huh, H. K. Kim, N. K. Kim, and Y. Lee, Basic examples and extensions of symmetric rings, J. Pure Appl. Algebra 202 (2005), no. 1-3, 154-167.

[12] C. Huh, H. K. Kim, and Y. Lee, p.p. rings and generalized p.p. rings, J. Pure Appl. Algebra 167 (2002), no. 1, 37-52.

[13] N. K. Kim and Y. Lee, Armendariz rings and reduced rings, J. Algebra 223 (2000), no. $2,477-488$ 
[14] _ Extensions of reversible rings, J. Pure Appl. Algebra 185 (2003), no. 1-3, 207223.

[15] R. L. Kruse and D. T. Price, Nilpotent Rings, Gordon and Breach, New York, London, Paris, 1969

[16] J. Lambek, Lectures on Rings and Modules, Blaisdell Publishing Company, Waltham, 1966.

[17] On the representation of modules by sheaves of factor modules, Canad. Math. Bull. 14 (1971), 359-368.

[18] G. Marks, Reversible and symmetric rings, J. Pure Appl. Algebra 174 (2002), no. 3, 311-318.

[19] L. Motais de Narbonne, Anneaux semi-commutatifs et unisériels anneaux dont les idéaux principaux sont idempotents, In: Proceedings of the 106th National Congress of Learned Societies (Perpignan, 1981), 71-73, Bib. Nat., Paris 1982.

[20] G. Shin, Prime ideals and sheaf representation of a pseudo symmetric ring, Trans. Amer. Math. Soc. 184 (1973), 43-60.

[21] W. Xue, Structure of minimal noncommutative duo rings and minimal strongly bounded nonduo rings, Comm. Algebra 20 (1992), no. 9, 2777-2788.

[22] L. Xu and W. Xue, Structure of minimal non-commutative zero-insertive rings, Math. J. Okayama Univ. 40 (1998), 69-76.

NAM KYUN KIM

Faculty of Liberal Arts and Sciences

HANBAT NATIONAL UNIVERSiTy

DAEJEON 305-719, KoREA

E-mail address: nkkim@hanbat.ac.kr

YANG LEE

Department of Mathematics Education

Pusan National University

Pusan 609-735, Korea

E-mail address: ylee@pusan.ac.kr

YEONSOOK SEO

Department of Mathematics

Pusan National University

Pusan 609-735, Korea

E-mail address: ysseo0305@pusan.ac.kr 